\title{
El seguimiento telefónico mejora la adherencia y reduce la mortalidad en pacientes polimedicados
}

\author{
Telephone counselling improves adhesion and reduces mortality in patients with polypharmacy
}

Wu J, y col. BMJ 2006; 333:522-527.

\section{Objetivo}

Evaluar el impacto del seguimiento telefónico periódico realizado por un farmacéutico, sobre la mortalidad y adherencia al tratamiento farmacológico de pacientes polimedicados.

Diseño

Ensayo clínico controlado aleatorizado de dos años de seguimiento.

\section{Lugar}

Centros Ambulatorios del Hospital Prince of Wales, Hong Kong.

\section{Pacientes}

De 1011 pacientes medicados con cinco o más fármacos, fueron considerados adherentes a un fármaco quienes tomaban 80 a $120 \%$ de la dosis diaria prescripta. La adherencia global se calculó dividiendo el número de fármacos a los que el paciente era adherente, por el número total prescripto, expresándose como porcentaje. De 502 individuos no adherentes, 442 aceptaron participar del estudio. La asignación fue ciega, aleatorizandose al grupo intervención a recibir seguimiento farmacéutico telefónico y al grupo control, cuidado convencional. Fueron excluidos los sujetos con trastornos psicológicos, demencia y/o problemas de comprensión idiomática, y los residentes en hogares para ancianos.

\section{Intervención}

Todos recibieron una charla educativa sobre el correcto uso de medicamentos de 10 a 15 minutos de duración dictada por un farmacéutico. Los del grupo "intervención" recibieron seis a ocho llamados telefónicos en el período comprendido entre las consultas al centro ambulatorio. El farmacéutico indagó sobre el tratamiento farmacológico, recalcó la importancia de la adherencia al mismo, recordó próximas consultas, aclaró dudas y explicó aspectos relevantes sobre dieta, ejercicio y automonitoreo.

\section{Medición de resultados}

Tiempo trascurrido hasta la muerte por cualquier causa, cambio en la adherencia y asociación entre adherencia y mortalidad.

\section{Resultados principales}

El consejo telefónico se asoció significativamente en forma inversa al riesgo de muerte. Ver tabla 1.

Tabla 1: efecto del monitoreo telefónico sobre la mortalidad y la adherencia a la toma de medicación en pacientes polimedicados.

\begin{tabular}{|l|c|c|c|c|}
\hline Mortalidad por & $\begin{array}{c}\text { Grupo } \\
\text { intervención }\end{array}$ & Grupo control & $\begin{array}{c}\text { RRR } \\
\text { (IC95\%) }\end{array}$ & NNT \\
\hline todas las causas & $25(11 \%)$ & $38(17 \%)$ & $41 \%(0,35 \mathrm{a} 0,97)$ & 16 \\
\hline
\end{tabular}

RRR: reducción del riesgo relativo. NNT: número necesario a tratar.

En la cohorte basal de 1011 pacientes y tomando como referencia al subgrupo con un puntaje de adherencia de $67 \%$ $(R R=1)$ el riesgo relativo de muerte fue 1,61 (IC95\% 1,05 a 2,48 ) en el subgrupo de "adherencia intermedia" (puntaje entre 34 y $66 \%$ ) y de 2,87 (IC95\% 1,80 a 2,57) en el subgrupo de menor adherencia (puntaje menor a 33\%). Al finalizar el seguimiento los pacientes intervenidos tendieron a permanecer más adherentes que los controles.

\section{Conclusiones}

En pacientes polimedicados, una baja adherencia al tratamiento farmacológico se asoció con un incremento de la mortalidad. El seguimiento telefónico por un farmacéutico mejoró la adherencia y redujo la mortalidad.

Palabras clave: polifarmacia, adherencia, seguimiento farmacéutico telefónico, ensayo clínico aleatorizado.

Key words: polypharmacy, compliance, pharmaceutical telephone counseling, randomized controlled trial.

Fuente de financiamiento/conflicto de interés de los autores: Hong Kong Fuente de financiamiento/conflicto de interes de los autores: Hong Kong
Governement Health Cared and Promotion Fund y Merck Sharp y Dome (MSD). Dos autores participan en programas de investigación de MSD.

\section{Comentario}

En las personas con enfermedades crónicas, la polimedicación se asocia a una baja adherencia y a un peor pronóstico, ${ }^{1,2}$, existiendo documentación bibliográfica ${ }^{3,4,5}$ de intervenciones farmacéuticas que pueden mejorar la adherencia y consecuentemente, la efectividad del tratamiento. Este estudio involucro pacientes de alto riesgo -antecedentes de visitas a guardias e internaciones en los años previos- con un promedio de consumo de 14 comprimidos por día y en el contexto de un sistema de salud con escaso rol del médico de cabecera, alta rotación de pacientes y pobre seguimiento longitudinal. No se evaluó el impacto sobre la calidad de vida ni el grado de satisfacción, y la intervención telefónica incluyó recordatorios de citas y pautas no farmacológicas, que podrían explicar parte de la mejora en los resultados.

\section{Conclusiones de los comentadores}

La incorporación del farmacéutico fue útil para el seguimiento y el cuidado de pacientes crónicos, pudiendo mejorar los resultados de los tratamientos indicados y optimizar la utilización de los recursos del sistema de salud. Consideramos ideal incluirla dentro de un programa de cuidados multidisciplinarios con registro único de la evolución de los pacientes y fluida comunicación entre los distintos profesionales intervinientes.

Leonardo Garfi [ Servicio de Clínica Médica. Hospital Italiano de Buenos Aires. leonardo.garfi@hospitalitaliano.org.ar ]

Natalia Korzewycz, Gabriela González Capdevila [ Farmacéuticas, Area de Farmacovigilancia. Hospital Italiano de Buenos Aires. gabriela.capdevila@hospitalitaliano.org.ar ] Recibido el 29/11/06 y aceptado el 01/05/2008

Garfi L, Korzewycz N, Gonzalez G. El seguimiento telefónico mejora la adherencia y reduce la mortalidad en pacientes polimedicados. Evid. actual. práct. ambul; 11(3):78, May-Jun 2008. Comentado de: Wu J Y F, Leung W Y S, Chang S y col. Effectiveness of telephone counselling by a pharmacist in reducing mortality in patients receiving polypharmacy: randomised controlled trial. BMJ 2006 Aug 17;333:522-527. BMJ, doi:10.1136/bmj.38905.447118.2F. PMID: 16916809. Disponible en: http://www.bmj.com/cgi/reprint/333/7567/522

\section{Referencias}

1. World Health Organization. Defining adherence. In: Adherence to long-term therapies: evidence for action, Geneva: WHO, 2003:3-5. Disponible en: www.who.int/chronic_conditions/adherencereport/en/

2. Haynes R y col. Systematic review of randomized trials of interventios to assist patients to follow prescrptions for medications. Lancet 1996; 348:383-6.

3. Friedman R, et al. A telecomunications system for monitoring and counseling patients with hypertension. Impact on medication adherence and blood pressure control. Am $\mathrm{J}$ Hypertens 1996;9: 285-92.

4. Guthrie R. The effects of postal and telephone reminders on compliance with pravastatine therapy in a national registry: results of the first myocardial infarction risk reduction program. Clin Ther 2001; 23:970-80.

5 . Leung $\mathrm{W}$ y col. The effects of struxtured care by a pharmacist- diabetes specialist team on renal outcomes in type 2 diabetic patients with nephropathy and renal impairment. Am J Med 2005; 118:1414. 